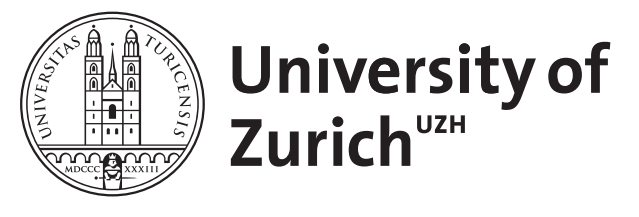

\title{
Tokolyse bei vorzeitiger Wehentätigkeit
}

Beinder, E ; Vetter, K

\begin{abstract}
Im September 2006 wurde die Leitlinie 015/025 "Medikamentöse Wehenhemmung bei drohender Frühgeburt" (http://www.dggg.de, http://www.awmf-online.de) publiziert. Sie zeigt den Korridor auf, innerhalb dessen die Verwendung von Tokolytika bei vorzeitiger Wehentätigkeit dem derzeitigen Stand der Wissenschaft und der sog. guten klinischen Praxis entspricht. Fenoterol, Atosiban, Indometacin und Nifedipin sind äquieffektiv, unterscheiden sich aber im Nebenwirkungsprofil, Kontraindikationen, Kosten und Zulassung. Erläutert werden die Empfehlungen, zusammengefasst werden die wesentlichen Ziele der medikamentösen Wehenhemmung: Durchführung der Lungenreifeprophylaxe bei drohender Frühgeburt, Schwangerschaftsverlängerung bei vorübergehenden Ursachen vorzeitiger Wehentätigkeit (z.B. Durchführung einer Cerclage, Pyelonephritis) sowie Ermöglichung des Transportes der Schwangeren in ein Perinatalzentrum
\end{abstract}

DOI: https://doi.org/10.1007/s00129-007-1963-1

Other titles: Tocolysis in cases of premature contractions

Posted at the Zurich Open Repository and Archive, University of Zurich

ZORA URL: https://doi.org/10.5167/uzh-155751

Journal Article

Published Version

Originally published at:

Beinder, E; Vetter, K (2007). Tokolyse bei vorzeitiger Wehentätigkeit. Der Gynäkologe, 40(4):279-289. DOI: https://doi.org/10.1007/s00129-007-1963-1 
Gynäkologe 2007 · 40:279-289

DOI 10.1007/s00129-007-1963-1

Online publiziert: 14. März 2007

(c) Springer Medizin Verlag 2007

\section{Redaktion}

D. Berg, Amberg (Schriftleitung)

R. Kreienberg, Ulm

T. Strowitzki, Heidelberg

K. Vetter, Berlin

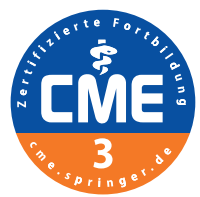

\section{CME.springer.de -}

\section{Zertifizierte Fortbildung für Kliniker} und niedergelassene Ärzte

Die CME-Teilnahme an diesem Fortbildungsbeitrag erfolgt online auf CME.springer.de und ist Bestandteil des Individualabonnements dieser Zeitschrift. Abonnenten können somit ohne zusätzliche Kosten teilnehmen.

Unabhängig von einem Zeitschriftenabonnement ermöglichen Ihnen CME.Tickets die Teilnahme an allen CME-Beiträgen auf CME.springer.de. Weitere Informationen zu CME.Tickets finden Sie auf CME.springer.de.

\section{Registrierung/Anmeldung}

Haben Sie sich bereits mit Ihrer Abonnementnummer bei CME.springer.de registriert? Dann genügt zur Anmeldung und Teilnahme die Angabe Ihrer persönlichen Zugangsdaten. Zur erstmaligen Registrierung folgen Sie bitte den Hinweisen auf CME.springer.de.

\section{Online teilnehmen}

\section{und 3 CME-Punkte sammeln}

Die CME-Teilnahme ist nur online möglich. Nach erfolgreicher Beantwortung von mindestens 7 der $10 \mathrm{CME}$-Fragen senden wir Ihnen umgehend eine Bestätigung der Teilnahme und der $3 \mathrm{CME}$ Punkte per E-Mail zu.

\section{Zertifizierte Qualität}

Diese Fortbildungseinheit ist zertifiziert von der Landesärztekammer Hessen und der Nordrheinischen Akademie für Ärztliche Fort- und Weiterbildung und damit auch für andere Ärztekammern anerkennungsfähig.

Folgende Maßnahmen dienen der Qualitätssicherung aller Fortbildungseinheiten auf CME.springer.de: Langfristige Themenplanung durch erfahrene Herausgeber, renommierte Autoren, unabhängiger Begutachtungsprozess, Erstellung der CME-Fragen nach Empfehlung des IMPP mit Vorabtestung durch ein ausgewähltes Board von Fachärzten.

Für Fragen und Anmerkungen stehen wir Ihnen jederzeit zur Verfügung:

Springer Medizin Verlag GmbH

Fachzeitschriften Medizin/Psychologie

CME-Helpdesk, Tiergartenstraße 17

69121 Heidelberg

E-Mail: cme@springer.com

CME.springer.de

\author{
E. Beinder ${ }^{1} \cdot$ K. Vetter $^{2}$ \\ ${ }^{1}$ Department Frauenheilkunde, UniversitätsSpital Zürich, Zürich \\ ${ }^{2}$ Klinik für Geburtsmedizin, Vivantes Klinikum Neukölln, Berlin
}

\section{Tokolyse bei vorzeitiger Wehentätigkeit}

\section{Zusammenfassung}

Im September 2006 wurde die Leitlinie 015/025 „Medikamentöse Wehenhemmung bei drohender Frühgeburt" (http://www.dggg.de, http://www.awmf-online.de) publiziert. Sie zeigt den Korridor auf, innerhalb dessen die Verwendung von Tokolytika bei vorzeitiger Wehentätigkeit dem derzeitigen Stand der Wissenschaft und der sog. guten klinischen Praxis entspricht. Fenoterol, Atosiban, Indometacin und Nifedipin sind äquieffektiv, unterscheiden sich aber im Nebenwirkungsprofil, Kontraindikationen, Kosten und Zulassung. Erläutert werden die Empfehlungen, zusammengefasst werden die wesentlichen Ziele der medikamentösen Wehenhemmung: Durchführung der Lungenreifeprophylaxe bei drohender Frühgeburt, Schwangerschaftsverlängerung bei vorübergehenden Ursachen vorzeitiger Wehentätigkeit (z. B. Durchführung einer Cerclage, Pyelonephritis) sowie Ermöglichung des Transportes der Schwangeren in ein Perinatalzentrum.

\section{Schlüsselwörter}

Vorzeitige Wehen · Tokolyse · Frühgeburt

\section{Tocolysis in cases of premature contractions}

\begin{abstract}
In September 2006, the German Guidelines 015/025 on tocolysis "Medikamentöse Wehenhemmung bei drohender Frühgeburt" (Pharmacological inhibition of contractions for threatened premature birth, http://www.dggg.de, http://www.awmf-online.de) were published. These guidelines were designed to aid obstetricians in the decision of when und how to use tocolytics to prevent preterm labour based on scientific knowledge and good clinical practice. Fenoterol, atosiban, indomethacin and nifedipine are equi-effective, but differ with respect to side effects for both mother and fetus, contraindications, cost, and not all are licensed for use in pregnancy. This article comments on the recommendations made in these guidelines and explains the main goals when treating preterm labour: administration of antenatal glucocorticoids to prevent neonatal respiratory distress syndrome, prolongation of pregnancy if self-limiting causes of preterm labour are present (e.g. during elective cerclage or pyelonephritis), and enabling transport of the pregnant woman into a perinatal care center.
\end{abstract}

\section{Keywords}

Preterm birth · Tocolysis · Premature birth 
Weltweit nimmt die Frühgeburtenrate nicht $a b$, sondern steigt eher an

Die perinatale Mortalität konnte insgesamt drastisch vermindert werden

Nur in etwa 30\% der Fälle von drohender Frühgeburt ist eine Tokolyse angezeigt
Die Ursachen vorzeitiger Wehentätigkeit sind mannigfaltig und umfassen aszendierende Infektionen, Mehrlingsschwangerschaften, fetale Ursachen und Uterusfehlbildungen. Eine monokausale Therapie der drohenden Frühgeburt kann es somit nicht geben. Vor Anwendung einer medikamentösen Tokolyse müssen Kontraindikationen sorgfältig ausgeschlossen werden. In der Regel wird eine medikamentöse Tokolyse bei idiopathischer Wehentätigkeit zwischen der 24. und 34. SSW für maximal 48 Stunden durchgeführt. Der Nutzen einer Dauertokolyse, einer Tokolyse vor 24 SSW, einer Kombination von Tokolytika, absoluter Bettruhe oder der simultanen Verabreichung von Gestagenen ist nicht nachgewiesen und sollte deshalb nur in ausgewählten Einzelfällen erfolgen. Bei der Auswahl der Tokolytika müssen Verträglichkeit, Zulassung, Erfahrung des Anwenders und auch ökonomische Aspekte berücksichtigt werden. Ein eindeutiges First-line-Tokolytikum gibt es derzeit nicht.

\section{Vorzeitige Wehen als Ursache von Frühgeburten}

In den meisten westeuropäischen Ländern beträgt die Frühgeburtenrate 7-8\%. Etwa 1\% aller Neugeborenen werden vor 32+0 SSW geboren, wenn die perinatale Morbidität und Mortalität besonders hoch sind. Es kann beobachtet werden, dass die Frühgeburtenrate weltweit nicht abnimmt, sondern eher ansteigt. Demgegenüber steht die Entwicklung der perinatalen Mortalität, die insgesamt drastisch vermindert werden konnte - insbesondere wenn Kollektive mit gleichen Ausgangsbedingungen, wie Gestationsalter oder Geburtsgewicht, miteinander verglichen werden. Der bayerischen Perinatalerhebung zufolge nahm die perinatale Mortalität in Bayern im Zeitraum von 1979 bis 2004 von 11 auf $4,8 \%$ ab.

Im Einzelnen können folgende Hauptkategorien der drohenden Frühgeburt unterschieden werden:

- Infektion: systemisch, aszendierend oder als Chorioamnionitis

- Fetale Ursachen wie Fehlbildungen mit Hydramnion und Mehrlinge

- Uterusanomalien: Myome, Uterusfehlbildungen und Zervixinsuffizienz

- Idiopathisch

- Plazentalokalisations- und Haftungsstörungen: Plazenta praevia, Plazentarandblutung und Plazentalösung

- Störungen der Plazentation und der Entwicklung der Plazenta mit der Folge einer Plazentainsuffizienz

- Mütterliche Ursachen: fieberhafte und andere Erkrankungen, Diabetes oder Präeklampsie.

Nur in etwa 30\% der Fälle von drohender Frühgeburt ist eine Tokolyse angezeigt (vor allem bei idiopathischer Wehentätigkeit).

\section{Untersuchungen vor Durchführung einer medikamentösen Tokolyse}

- Kardiotokographie zur Beurteilung der Häufigkeit von Kontraktionen und des fetalen Zustandes

- Spekulumuntersuchung mit Bestimmung des vaginalen $\mathrm{pH}$-Wertes, Entnahme bakteriologischer Abstriche einschließlich Chlamydienabstrich (wenn keine Urin-PCR durchgeführt wurde) und Beurteilung der Zervixlänge. Auf eine Palpation der Zervix sollte insbesondere bei Verdacht auf einen Blasensprung verzichtet werden.

- Mit einem negativen Fibronektintest können Schwangere mit einem aktuell nicht erhöhten Risiko für eine Frühgeburt identifiziert werden.

- Vaginalsonographische Messung der Zervixlänge: Vor 32 SSW ist eine Zervixlänge von <25 mm als verkürzt anzusehen.

- Abdominalsonographie mit Fetometrie, Fruchtwassermengenbestimmung und Doppler-Sonographie der uteroplazentofetalen Einheit sowie Ausschluss fetaler Fehlbildungen und einer Mehrlingsschwangerschaft (v.a. Ausschluss eines fetofetalen Transfusionssyndroms).

- Ausschluss einer systemischen Infektion durch klinische Untersuchung, Temperaturmessung, Urinstix und Laboruntersuchungen (Leukozytenzahl, CRP-Konzentration). 
Abb. 1 Vorgehen bei vorzeitigen Kontraktionen

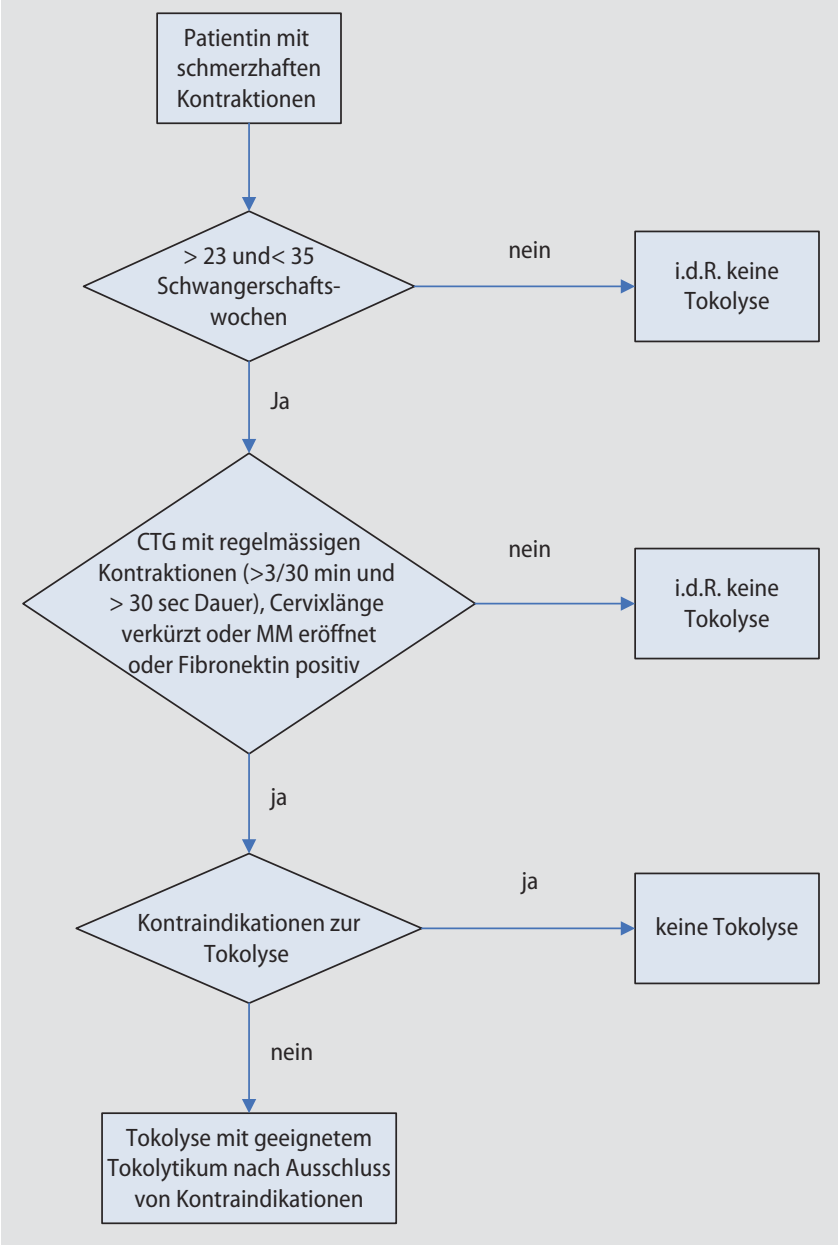

Eine Übersicht zum Vorgehen bei vorzeitigen Wehen gibt $\bullet$ Abb. 1.

\section{Definition vorzeitiger Wehen}

Es gibt keine auf eindeutiger wissenschaftlicher Evidenz basierende Definition der vorzeitigen Wehentätigkeit, und auch nicht darüber, wann eine medikamentöse Tokolyse eindeutig von Nutzen zur Schwangerschaftsverlängerung ist. In Terminnähe gebären 50-70\% aller Schwangeren mit der klinischen Diagnose vorzeitige Wehentätigkeit, die mit Placebo behandelt werden [10]. Die besten derzeit bekannten prädiktiven Marker für effektive vorzeitige Wehen sind der vaginalsonographische Nachweis einer progredienten Zervixverkürzung und ein positiver Fibronektintest im Vaginalsekret [6]. Fibronektin findet sich bei einer choriodezidualen Dissoziation in erhöhten Konzentrationen im Zervikalsekret und hat einen geringen positiven, aber einen hohen negativen Vorhersagewert für eine Frühgeburt. Der fehlende Nachweis von Fibronektin spricht also mit hoher Wahrscheinlichkeit für ein geringes Frühgeburtsrisiko.

Eine medikamentöse Tokolyse sollte deshalb in der Regel nur dann erfolgen, wenn

- eine signifikante vorzeitige Wehentätigkeit (schmerzhafte, palpable, länger als $30 \mathrm{~s}$ andauernde Kontraktionen, die häufiger als 3-mal pro 30 min auftreten) und

- eine funktionelle Zervixverkürzung zusammentreffen und/oder

- eine Muttermunderweiterung nachweisbar ist und/oder

- der Fibronektintest im Vaginalsekret positiv ausfällt.

Im Einzelfall oder in Hochrisikosituationen - wie z. B. bei höhergradigen Mehrlingen oder einer Blutung bei $>$ Placenta praevia- kann von diesen Kriterien abgewichen und nach individueller klinischer Evaluation bereits bei Nachweis regelmäßiger Kontraktionen eine Tokolyse eingeleitet werden.
- Choriodeziduale Dissoziation

Der fehlende Nachweis von Fibronektin spricht mit hoher Wahrscheinlichkeit für ein geringes Frühgeburtsrisiko

\section{Placenta praevia}


Eine Tokolyse ist absolut kontraindiziert, wenn sie für Mutter und/oder Feten schädlich wäre

Schon nach der 34+0. SSW bestehen nur noch geringe frühgeburtsbedingte Risiken für das Neugeborene

Vor der 24+0. SSW ist die Einleitung einer medikamentösen Tokolyse in der Regel nicht indiziert

\section{Pyelonephritis}

Auch ohne erkennbare Pathologie gelingt mit der Tokolyse nicht sicher eine Verlängerung der Schwangerschaft um mehr als 2-7 Tage gegenüber Placebo

\section{Lungenreifeinduktion}

\section{Allgemeine Kontraindikationen}

Eine Tokolyse ist absolut kontraindiziert, wenn sie für Mutter und/oder Feten schädlich wäre. Dies ist der Fall bei

- intrauteriner Infektion,

- nicht mit dem Leben vereinbarer Fehlbildung des Feten oder intrauterinem Fruchttod,

- mütterlicher Indikation zur Schwangerschaftsbeendigung, vor allem vaginalen Blutungen mit hämodynamischer Instabilität und schwere Präeklampsie/Eklampsie, sowie bei

- kindlicher Indikation zur Schwangerschaftsbeendigung mit Ausnahme der Akuttokolyse zur kurzzeitigen Überbrückung einer fetalen Notsituation.

Der Vorteil einer Tokolyse ist in folgenden Situationen nicht nachgewiesen und Nachteile können für Mutter und Feten auftreten, sodass nur in begründeten Einzelfällen eine Tokolyse durchgeführt werden sollte: Schwangerschaftsalter $>34+0$ Wochen und schwere intrauterine Wachstumsrestriktion des Feten.

\section{Wann kann eine medikamentöse Tokolyse sinnvoll eingesetzt werden?}

Obwohl eine Frühgeburt als Entbindung vor der abgeschlossenen 37 SSW (37+0) definiert ist, bestehen bereits nach $34+0$ SSW nur noch geringe frühgeburtsbedingte Risiken für das Neugeborene in Form von schwerer Morbidität und Mortalität. Eine medikamentöse Tokolyse ist aufgrund der Nebenwirkungen für die Mutter und des ungünstigen Nutzen-Risiko-Verhältnisses für den Feten nach $34+0$ SSW in der Regel nicht mehr indiziert. Seltene individuelle Ausnahmen (z. B. Verlegung in ein perinatologisches Zentrum bei zusätzlichen fetalen Problemen) können bestehen.

Der früheste Zeitpunkt der Einleitung einer Tokolyse bei vorzeitiger Wehentätigkeit ist umstritten, da es keine randomisierten Untersuchungen zu diesem Thema gibt [7]. Der Haupteffekt der Tokolyse besteht in einer Verlängerung der Schwangerschaft um 2-7 Tage gegenüber Placebo. Somit ist die Einleitung einer medikamentösen Tokolyse vor 24+0 SSW in der Regel nicht indiziert. Als Faustregel gilt, dass eine Tokolyse dann Sinn ergibt, wenn auch eine postnatale Therapie eingesetzt würde. Individuelle Ausnahmen können begründet vorliegen, vor allem, wenn eine vorübergehenden Ursache von vorzeitiger Wehentätigkeit besteht, z. B. eine Pyelonephritis oder eine intraabdominale Operation.

\section{Ergebnisse der Tokolyse}

Die mit der Einführung potenter Medikamente zur Hemmung von Kontraktionen des Myometriums verbundenen Erwartungen, wie eine substantielle Senkung der Frühgeburtenrate und damit der perinatalen Mortalität, haben sich nicht erfüllt. Dafür gibt es im Wesentlichen zwei Erklärungen: Der Anteil der Frühgeburten, bei denen eine Verlängerung der Schwangerschaft bis zu 37. SSW wünschenswert wäre, beträgt weniger als ein Drittel der Frühgeburten. Die Mehrzahl der prospektiv randomisierten Studien zeigt, dass bei vorzeitiger Wehentätigkeit auch ohne erkennbare mütterliche oder fetale Pathologie eine Verlängerung der Schwangerschaft um mehr als 2-7 Tage gegenüber Placebo nicht sicher gelingt.

Primäre Outcome-Variable war allerdings bei fast allen Untersuchungen zur Tokolyse die Verlängerung der Schwangerschaft um 48 Stunden. Keine der Studien hatte genügend statistische Power, um eine Reduktion von peri- oder neonataler Mortalität nachzuweisen. Zudem wurden nicht selten Patientinnen mit einem Schwangerschaftsalter von mehr als 32-34 SSW eingeschlossen, wenn die perinatale Morbidität und Mortalität bereits sehr gering sind. Die durchschnittliche Schwangerschaftsverlängerung von 2-7 Tagen gegenüber Placebo, die für $\beta$-Agonisten, Atosiban und COX(Cyclooxygenase)Hemmer gezeigt wurde, kann für die Durchführung der $>$ Lungenreifeinduktion mit Betamethason [2] und ggf. für den Transport in ein Perinatalzentrum genützt werden (Leitlinie „Antepartaler Transport von Risikoschwangeren“ Nr. 024/001 der Gesellschaft für Neonatologie und Pädiatrische Intensivmedizin). Unabhängig davon führt jeder Tag der Schwangerschaftsverlängerung im Zeitraum von 24 bis 26 SSW zu einer signifikant verbesserten neonatalen Überlebensrate. 
Tab. 1 Charakteristika gebräuchlicher Tokolytika

\begin{tabular}{|c|c|c|c|c|}
\hline Tokolytika & $\begin{array}{l}\text { Zugelassen zur } \\
\text { Tokolyse }\end{array}$ & $\begin{array}{l}\text { Wirksamkeit ge- } \\
\text { genüber Placebo }\end{array}$ & Nebenwirkungen & Administration \\
\hline$\beta$-Mimetika & $\begin{array}{l}\text { Fenoterol (Partusis- } \\
\text { ten }^{\circledast} \text { ) }\end{array}$ & Nachgewiesen & +++ & I.v. \\
\hline $\begin{array}{l}\text { Oxytocin-Rezepto- } \\
\text { rantagonisten }\end{array}$ & $\begin{array}{l}\text { Atosiban (Tracto- } \\
\text { cile }^{\oplus} \text { ) }\end{array}$ & Nachgewiesen & + & I.v. \\
\hline $\begin{array}{l}\text { Kalziumantagonis- } \\
\text { ten (Nifedipin) }\end{array}$ & Nein & Nicht untersucht & + bis ++ & P.o. \\
\hline Magnesium & $\begin{array}{l}\text { Formelle Zulassung } \\
\text { nicht erfolgt }\end{array}$ & Nicht nachgewiesen & +++ & I.v. \\
\hline Indometacin & Nein & Nachgewiesen & $\begin{array}{l}+ \text { (Mutter) bzw. } \\
+++ \text { (Fet) }\end{array}$ & P.o. \\
\hline NO-Donatoren & Nein & $\begin{array}{l}\text { Datenlage noch } \\
\text { nicht ausreichend }\end{array}$ & + bis ++ & Transdermal \\
\hline
\end{tabular}

\section{First-line-Tokolytikum}

\section{Effektivität}

Nach dem derzeitigen Wissensstand sind Atosiban, $\beta$-Mimetika, Nifedipin und Indometacin äquieffektiv in der Hemmung vorzeitiger Wehen. Weitere zur Tokolyse eingesetzte Substanzen sind Nitroglycerin und Magnesiumsulfat. (Auch Alkohol ist in sehr hohen Dosen nachgewiesenermaßen effektiv in der Hemmung vorzeitiger Wehen, wird allerdings aus offensichtlichen Gründen therapeutisch nicht mehr eingesetzt.)

\section{Zulassung}

Die Substanzen unterscheiden sich in ihrer Wirksamkeit, der Applikationsform, dem Nebenwirkungsprofil und den Kosten (• Tab. 1, 2, 3, 4). Nur der $\beta$-Agonist Fenoterol (Partusisten ${ }^{\bowtie}$ ), der Oxytocin-Rezeptorantagonist Atosiban (Tractocile ${ }^{\circledast}$ ) und Magnesium sind zur Behandlung vorzeitiger Wehen in Deutschland zugelassen. Für Nifedipin, Indometacin und Nitroglycerin wurde eine Zulassung in der Schwangerschaft nicht beantragt. Die World Health Organisation (WHO) führt Nifedipin allerdings wegen seiner quasi ubiquitären Verfügbarkeit einerseits und seiner nachgewiesenen Wirksamkeit andererseits als einziges „Antioxytocicum“ in der Liste der essenziellen Medikamente („essential medications“) auf. Die Zulassung von Atosiban wurde in den USA von der US Food and Drug Administration, FDA, abgelehnt, da sich in einer Untersuchung ein Trend zu einer höheren Rate an fetal-neonataler Sterblichkeit in der Atosibangruppe ergab [17].

\section{Empfehlungen internationaler Leitlinien}

In der Leitlinie des Royal College of Obstetricians and Gynaecologists (RCOG; Oktober 2002) werden $\beta$-Mimetika nicht mehr als Mittel erster Wahl zur Durchführung einer Tokolyse angesehen. Bevorzugt werden Nifedipin und Atosiban. Es wird jedoch darauf hingewiesen, dass Nifedipin zur Anwendung in der Schwangerschaft nicht zugelassen und Atosiban teuer ist. In den kanadischen Leitlinien wird Indometacin als Tokolytikum der ersten Wahl angegeben (Obstetric Guideline 2A des British Columbia Reproductive Care Program, BCRCP; Oktober 2001). In der amerikanischen (ACOG practice bulletin des American College of Obstetricians and Gynecologists Mai 2003) wird ebenso wie in der deutschen Leitlinie festgehalten, dass es kein eindeutiges First-line-Tokolytikum gibt und die Auswahl somit von den Erfahrungen und Abwägungen des anwendenden Arztes abhängt.

\section{Wirkungsmechanismus von Tokolytika}

$\beta$-Mimetika sind die am häufigsten verwendeten Medikamente zur Tokolyse. Die Wirkung der Schwangerschaftsprolongation mit $\beta$-Mimetika ist in randomisierten, placebokontrollierten Studien

Wegen seiner Verfügbarkeit und nachgewiesenen Wirksamkeit zählt Nifedipin als einziges „Antioxytocicum" zur WHO-Liste der „essential medications"

Es gibt kein eindeutiges First-lineTokolytikum 
Tab. 2 Nebenwirkungen einzelner Tokolytika

\begin{tabular}{|c|c|c|}
\hline & Für die Mutter & Für den Feten \\
\hline$\beta$-Mimetika & $\begin{array}{l}\text { Palpitationen (48\%), Tremor (39\%), Übelkeit } \\
(20 \%), \text { Kopfschmerz (23\%) und Thorax- } \\
\text { schmerzen (10\%). Schwere maternale Neben- } \\
\text { wirkungen - v.a. maternale Herzrhythmusstö- } \\
\text { rungen und Lungenödem -, die zu mütter- } \\
\text { lichen Todesfällen führten, sind beschrieben, } \\
\text { wobei das Lungenödem mit einer Häufigkeit } \\
\text { von etwa 1:425 (manchen Autoren zufolge } \\
\text { auch häufiger) auftritt [8]. Prädisponierende } \\
\text { Faktoren sind unkontrollierte übermäßige } \\
\text { Hydratation, gleichzeitige Präeklampsie } \\
\text { oder Lungenreifeinduktion, Bluttransfusion, } \\
\text { Mehrlingsschwangerschaften und maternale } \\
\text { Herzerkrankung. } \\
\text { Diabetogen } \\
\text { Die Bolustokolyse mit Fenoterol gilt als eine } \\
\text { nebenwirkungsärmere Alternative zur konti- } \\
\text { nuierlichen Infusion. }\end{array}$ & $\begin{array}{l}\text { Da } \beta \text {-Mimetika die Plazentaschranke passie- } \\
\text { ren, ähnlich wie bei der Mutter. } \\
\text { Diabetogen }\end{array}$ \\
\hline Atosiban & $\begin{array}{l}\text { Palpitationen (2\%), Tachykardie (6\%), Hypo- } \\
\text { tonie (3\%), Übelkeit (12\%), Erbrechen (7\%) } \\
\text { und Kopfschmerzen (10\%) [21] liegen nicht } \\
\text { über der Placebogruppe. Schwere Nebenwir- } \\
\text { kungen wurden unter alleiniger Therapie mit } \\
\text { Atosiban bisher nicht beschrieben. }\end{array}$ & $\begin{array}{l}\text { In einer Untersuchung Trend zu einer höheren } \\
\text { Rate an fetal-neonataler Sterblichkeit [17] }\end{array}$ \\
\hline $\begin{array}{l}\text { Nifedipin } \\
\text { (Slow-release) }\end{array}$ & $\begin{array}{l}\text { Übelkeit, Flush, Kopfschmerz, Tachykardie und } \\
\text { Palpitationen können auftreten. Diese Neben- } \\
\text { wirkungen sind in der Regel mild und weniger } \\
\text { schwer als die Nebenwirkungen, die unter } \\
\beta \text {-Mimetika gesehen werden. Blutdrucksen- } \\
\text { kung. Bei normotensiven Patienten sind die } \\
\text { kardiovaskulären Effekte gering. } \\
\text { Es wurden allerdings einzelne Fälle von } \\
\text { schweren Nebenwirkungen (Lungenödem, } \\
\text { Herzinfarkt, Hypoxie) beschrieben, fast immer } \\
\text { in Kombination mit der Verabreichung von } \\
\beta \text {-Mimetika und/oder sublingualer Verabrei- } \\
\text { chung mit schnellem Wirkungseintritt. }\end{array}$ & $\begin{array}{l}\text { Aus den bisherigen Publikationen ergeben } \\
\text { sich keine Hinweise für eine teratogene Po- } \\
\text { tenz oder eine bedeutsame Einschränkung } \\
\text { der uteroplazentaren Perfusion. In einer } \\
\text { Kasuistik wurde eine schwere maternale Hy- } \\
\text { potonie mit konsekutivem Tod des Feten be- } \\
\text { schrieben, nachdem Nifedipin gekaut worden } \\
\text { war und so eine sehr schnelle Wirkstoffverfüg- } \\
\text { barkeit vorhanden war [20]. }\end{array}$ \\
\hline Magnesium i.v. & $\begin{array}{l}\text { In einer höheren Dosierung mit erheblichen } \\
\text { maternalen Nebenwirkungen (Lungenödem, } \\
\text { Dyspnoe, Übelkeit, Erbrechen, Kopfschmerz, } \\
\text { Ateminsuffizienz) assoziiert, die ein Dauer- } \\
\text { monitoring der Mutter - insbesondere der } \\
\text { Atemfrequenz - erfordern }\end{array}$ & $\begin{array}{l}\text { V.a. Erhöhung der kindlichen Mortalität, wenn } \\
\text { sehr hohe Dosen angewendet werden („loa- } \\
\text { ding dose“ } 4-6 \mathrm{~g} \text { Magnesiumsulfat, Erhal- } \\
\text { tungsdosis 2-4 g/h über mehr als } 24 \mathrm{~h} \text { ) [4]. In } \\
\text { niedrigerer Dosierung (bis kumulativ maximal } \\
28 \mathrm{~g} \text { Magnesiumsulfat) zeigte das „Australasi- } \\
\text { an Collaborative Trial of Magnesium Sulphate“ } \\
\text { allerdings einen neuroprotektiven Effekt von } \\
\text { Magnesiumsulfat auf das fetale ZNS, ohne die } \\
\text { kindliche Mortalität signifikant zu beeinflus- } \\
\text { sen [3]. }\end{array}$ \\
\hline Indometacin & $\begin{array}{l}\text { Bei Beachtung der Kontraindikationen und } \\
\text { kurzer Behandlungsdauer gering }\end{array}$ & $\begin{array}{l}\text { Bei Anwendung }>48 \mathrm{~h} \text { sind persistierende } \\
\text { fetale Anurie, renale mikrozystische Läsionen } \\
\text { und neonatale Todesfälle beschrieben. Bei } \\
\text { Applikation von Indometacin über mehr als } \\
48 \text { Stunden nach } 32 \text { SSW muss in } 20-50 \% \\
\text { der Fälle mit einer Konstriktion bzw. einem } \\
\text { vorzeitigen Verschluss des D. arteriosus Botalli } \\
\text { gerechnet werden [15]. Die pränatale Kons- } \\
\text { triktion des D. arteriosus und eine Erhöhung } \\
\text { des Risikos für eine nekrotisierende Entero- } \\
\text { kolitis sind weitere Komplikationen für den } \\
\text { Feten bzw. das Neugeborene [16]. }\end{array}$ \\
\hline Nitroglycerin & $\begin{array}{l}\text { Erhebliche Kopfschmerzen und (ähnlich wie } \\
\text { bei Nifedipin) Gefahr der arteriellen Hypo- } \\
\text { tension }\end{array}$ & Nicht bekannt \\
\hline
\end{tabular}


Tab. 3 Kontraindikationen einzelner Tokolytika

$\beta$-Mimetika

Nifedipin („slow release")

Magnesium

Indometacin

Nitroglycerin

Tab. 4 Dosierung gängiger Tokolytika

\begin{tabular}{|c|c|}
\hline & Dosierung \\
\hline Fenoterol & $\begin{array}{l}\text { I.v. über Perfusor/Infusomat } \\
\text { Beginn: } 2 \mu \mathrm{g} / \mathrm{min} \text {. Steigerung um 0,8 } \mu \mathrm{g} \text { alle } 20 \mathrm{~min} \\
(4 \mu \mathrm{g} / \mathrm{min} \text { maximal) Bolustokolyse: Beginn: } 3-5 \mu \mathrm{g} \text { alle } \\
3 \mathrm{~min}\end{array}$ \\
\hline Atosiban & $\begin{array}{l}\text { I.v. über Perfusor/lnfusomat } \\
-6,75 \mathrm{mg} \text { über } 1 \mathrm{~min} \text { (Bolus) } \\
-18,00 \mathrm{mg} / \mathrm{h} \text { über } 3 \mathrm{~h}=300 \mu \mathrm{g} / \mathrm{min} \\
-6,00 \mathrm{mg} / \mathrm{h} \text { über } 15-45 \mathrm{~h}=100 \mu \mathrm{g} / \mathrm{min}\end{array}$ \\
\hline Nifedipin & $\begin{array}{l}\text { Oral } \\
\text { Die optimale Dosis von Nifedipin zur Behandlung vor- } \\
\text { zeitiger Wehen ist bisher nicht eindeutig definiert. Eine } \\
\text { gebräuchliche Dosierung ist die Verabreichung von } \\
\text { retardiertem Nifedipin (Nifedipin CR 30,60) in } 2 \text { oder } 3 \\
\text { Dosen, Höchstdosis: } 150 \mathrm{mg} / \mathrm{Tag}\end{array}$ \\
\hline Magnesium & $\begin{array}{l}\text { I.v. über Perfusor/Infusomat } \\
-4-6 \mathrm{~g} \text { als Bolus über } 15-30 \mathrm{~min} \\
-1-2(-3) \mathrm{g} / \mathrm{h}\end{array}$ \\
\hline Indometacin & $\begin{array}{l}\text { Oral/Rektal } \\
\text { Beginn: } 50 \mathrm{mg} \text {, Fortsetzung: } 25 \mathrm{mg} \text { alle } 4-6 \mathrm{~h} \\
\text { Rektal: } 100 \mathrm{mg} \text {, Fortsetzung oral: } 25 \mathrm{mg} \text { alle } 4-6 \mathrm{~h} \\
\text { (nicht länger als } 48 \mathrm{~h} \text {, nur bis } 32 \mathrm{SSW} \text { ) }\end{array}$ \\
\hline NO-Donatoren & $\begin{array}{l}\text { Transdermal } \\
\text { Die optimale Dosis von NO-Donatoren zur Behandlung } \\
\text { vorzeitiger Wehen ist nicht eindeutig definiert. } \\
\text { Bisher in Studien verwendete Dosierungen: } 1 \text {-2 Pflas- } \\
\text { ter mit } 10 \mathrm{mg} / \mathrm{Tag} \text { bis } 1-2 \text { Pflaster mit } 50 \mathrm{mg} / \mathrm{Tag}\end{array}$ \\
\hline
\end{tabular}

nachgewiesen worden. Die Wirkung wird über zellmembranständige $\beta$-adrenerge Rezeptoren vermittelt. $\beta$-Mimetika sind vor allem für die Mutter nebenwirkungsreich. Die Bolustokolyse mit Fenoterol gilt als eine nebenwirkungsärmere Alternative zur kontinuierlichen Infusion von Fenoterol.

Atosiban ist ein kompetitiver, nichtselektiver Antagonist für Oxytocin und den Arginin-Vasopressin-Rezeptor, der oxytocininduzierte Kontraktionen unterdrückt. Die Wirkung von Atosiban in der Schwangerschaftsprolongation wurde in einer randomisierten, placebokontrollierten Studien nachgewiesen [17]. Die Nebenwirkungen für die Mutter sind gering.

Nifedipin blockiert direkt den Influx von Kalziumionen durch die Zellmembran. Es gibt keine placebokontrollierten Studien, welche die Therapie vorzeitiger Wehen durch Nifedipin untersuchten. Die Einstufungen der Wertigkeit von Kalziumantagonisten zur Wehenhemmung basieren auf vergleichenden Untersuchungen mit anderen Tokolytika (v.a. Ritodrin). Die Verträglichkeit des Medikamentes ist bei der Verwendung von $>$ Slow-release-Präparaten und unter Ausschluss von Kontraindikationen gut.
$\beta$-Mimetika sind vor allem für die Mutter nebenwirkungsreich 
Magnesiumsulfat wird in einer Review der Cochrane Database als ineffektiv beschrieben

- Stickstoffmonoxid-(NO-)Donator

Eine Dauertokolyse sollte nur in Einzelfällen erfolgen

Die Kombination von Tokolytika ist ungenügend untersucht und daher nicht angezeigt
Indometacin inhibiert Zyklooxygenaseenzyme, die für die Synthese von Prostaglandinen verantwortlich sind. Es liegt eine einzelne placebokontrollierte Studie vor, außerdem 3 Studien zu Indometacin im Vergleich zu $\beta$-Agonisten, die bei sehr kleinen Untersuchungszahlen die tokolytische Wirksamkeit nachweisen. Die Verträglichkeit für die Mutter ist gut, die für den Feten nach einer Anwendungsdauer von mehr als 48 Stunden schlecht.

Magnesium wirkt intrazellulär als Kalziumantagonist. Interessanterweise gibt es keine einzige tragfähige wissenschaftliche Untersuchung, die eine tokolytische Wirksamkeit von Magnesium nachweist. Die einzige randomisierte, kontrollierte Untersuchung von Magnesiumsulfat gegenüber Placebo erbrachte keinen Wirkungsnachweis [1]. Magnesiumsulfat wird in einer Review der Cochrane Database als ineffektiv in der Verzögerung der Geburt oder in der Verhinderung der vorzeitigen Geburt beschrieben [4]. Die vorliegenden Untersuchungen wurden zum Teil in Dosen von bis zu $4 \mathrm{~g} /$ h durchgeführt und sind mit ähnlich gravierenden Nebenwirkungen für die Mutter assoziiert wie $\beta$ Mimetika. Es gibt ernst zu nehmende Hinweise darauf, dass Magnesium in hohen Dosen (2-4 g/h) und längerer Anwendungszeit mit einer erhöhten kombinierten fetalen, neonatalen und postneonatalen Mortalität assoziiert ist.

Nitroglycerin ist eine neuere und in Zukunft möglicherweise interessante Substanz zur Tokolyse. Nitroglycerin wirkt als $>$ Stickstoffmonoxid-(NO-)Donator und führt zur Muskelrelaxation. In der Cochrane Analyse von 2003, die allerdings nur 5 der 10 bisher publizierten Studien einbezog, wurde trotz eines günstigeren Nebenwirkungsprofils der Einsatz von Nitroglycerinpflastern aufgrund einer noch nicht ausreichenden Datenlage nicht zur Routineanwendung empfohlen [5].

\section{Indikationen für eine Dauertokolyse}

Auch nach initialem Ansprechen auf die Tokolysetherapie haben viele Frauen weiterhin ein hohes Risiko für eine vorzeitige Wehentätigkeit. Für die Erhaltungstherapie mit Atosiban gibt es eine placebokontrollierte Untersuchung, die bei der kontinuierlichen subkutanen Verabreichung eine Dauer bis zum Wiederauftreten von Wehen von 33 Tagen gegenüber 27 Tagen in der Placebogruppe feststellte [19]. Für alle anderen Tokolytika gibt es keine randomisierten Studien zum Nutzen einer Dauertokolyse. Im klinischen Alltag gibt es bei einzelnen Patientinnen Konstellationen, bei denen auf die Fortführung der Tokolyse über 48 Stunden hinaus schwerlich verzichtet werden kann.

Aufgrund der beschränkten Studienlage besteht derzeit keine Evidenz dafür, dass eine Dauertokolyse effektiver ist als eine kurz dauernde Tokolyse über maximal 48 h. Eine Dauertokolyse sollte somit nur in ausgewählten Einzelfällen erfolgen, z. B. bei symptomatischer Placenta praevia in einem frühen Schwangerschaftsstadium.

\section{Kombination von Tokolytika}

Auch unter Beachtung der substanzspezifischen Kontraindikationen sind schwerwiegende mütterliche Komplikationen durch die alleinige Anwendung von $\beta$-Mimetika, Nifedipin und Magnesiumsulfat in hoher Dosierung beschrieben worden. Nifedipin kann schwere Komplikationen verursachen, wenn es in Kombination mit oder nach Gabe von $\beta$-Mimetika und/oder hochdosiertem Magnesiumsulfat verabreicht wird. Die pharmakokinetischen und pharmakodynamischen Daten in der Schwangerschaft sind für die meisten zur Tokolyse verwendeten Substanzen unbekannt. Die Kombination von Tokolytika ist ungenügend untersucht und ihre Nebenwirkungen nach dem derzeitigen Wissen unabsehbar. Die Kombination von Tokolytika ist deshalb nicht angezeigt.

\section{Orale Tokolyse}

Für die Tokolytika Nifedipin und Indometacin ist ein Wirksamkeitsnachweis bei oraler Einnahme gegenüber Placebo nachgewiesen.

Es gibt mehrere Untersuchungen, die keine tokolytische Aktivität von oral verabreichtem Magnesium nachweisen konnten.

Für oral verabreichte $\beta$-Mimetika wurden in randomisierten Untersuchungen widersprüchliche Ergebnisse gewonnen: Während eine prospektiv randomisierte, doppelt verblindete Untersuchung einen günstigen Effekt von oralem Ritodrin in einer Slow-release-Form nachwies [9], erbrachte die 
Mehrzahl der Untersuchungen keinen Vorteil. Aus diesem Grund erscheint eine orale Tokolyse mit $\beta$-Mimetika oder Magnesium als nicht sinnvoll.

\section{Additive Maßnahmen}

Entsprechend den Indikationen zur Verlegung in ein perinatologisches Zentrum anhand der Leitlinie „Antepartaler Transport von Risikoschwangeren“ (Nr. 024/001) der Gesellschaft für Neonatologie und Pädiatrische Intensivmedizin sollte der Zeitraum bis zur Entbindung zur Verlegung der Schwangeren in ein Perinatalzentrum genutzt werden. Die Zeit sollte auch genutzt werden, um einen einzelnen Zyklus von Betamethason (2-mal $12 \mathrm{mg}$ i.m. im Abstand von $24 \mathrm{~h}$ ) zur Induktion der fetalen Lungenreife zu verabreichen [2].

Die routinemäßige Anwendung von Antibiotika bei erhaltener Fruchtblase aus dem alleinigen Grund der Prävention einer Frühgeburt ist dagegen nicht indiziert [11, 12, 13].

Ebenso sind Maßnahmen wie absolute Bettruhe, Hydratation, Sedierung und die Verabreichung von Gestagenen im Regelfall nicht angezeigt [14, 18].

Wenn eine Patientin mit vorzeitiger Wehentätigkeit stationär behandelt wird, dann ist auch eine engmaschige fetale Überwachung mit Kardiotokographie und Sonographie (Biometrie und Doppler-Sonographie) zu empfehlen.

\section{Korrespondierender Autor PD Dr. E. Beinder}

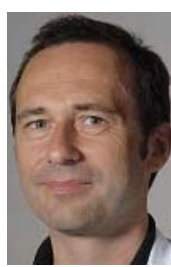

Department Frauenheilkunde, Universitätsspital Zürich

Frauenklinikstr. 10, 8091 Zürich, Schweiz

ernst.beinder@usz.ch

Interessenkonflikt. Es besteht kein Interessenkonflikt. Der korrespondierende Autor versichert, dass keine Verbindung mit einer Firma, deren Produkt in dem Artikel genannt oder einer Firma, die ein Konkurrenzprodukt betreibt, bestehen. Die Präsentation des Themas ist unabhängig und die Darstellung der Inhalte produktneutral.

\section{Literatur}

1. Cox SM, Sherman ML, Leveno KJ (1990) Randomized investigation of Magnesium sulfate for prevention of preterm birth. Am J Obstet Gynecol 163: 767-772

2. Crowley P (2000) Prophylactic corticosteroids for preterm birth. Cochrane Database Syst Rev 2: CD000065

3. Crowther CA, Hiller JE, Doyle LW, Haslam RR (2003) Effect of magnesium sulphate given for neuroprotection before preterm birth. JAMA 290: 2669-2676

4. Crowther CA, Hiller JE, Doyle LW (eds) (2004) Magnesium sulphate for preventing preterm birth in threatened preterm labour (Cochrane Review). The Cochrane Library, Issue 1, 2004. John Wiley, Chichester, UK

5. Duckitt K, Thornton S (2002) Nitric oxide donors for the treatment of preterm labour. Cochrane Database Syst Rev 2002: CD002860
6. Goldenberg RL, Mercer BM, lams JD et al. (1997) The preterm prediction study: patterns of cervicovaginal fetal fibronectin as predictors of spontaneous preterm delivery. National Institute of Child Health and Human Development Maternal-Fetal Medicine Units Network. Am J Obstet Gynecol 177: 8-12

7. Goldenberg RL (2002) The management of preterm labor. Obstet Gynecol 100: 1020

8. Gyetvai K, Hannah ME, Hodnett ED, Ohlsson A (1999) Tocolytics for preterm labor: a systematic review. Obs tet Gynecol 94: 869-877

9. Holleboom CA, Merkus JM, Elferen LW van, Keirse MJ (1996) Doubleblind evaluation of ritodrine sustained release for oral maintenance of tocolysis after active preterm labour. Br J Obstet Gynaecol 103: 702 705

10. Jeyabalan A, Caritis SN (2002) Pharmakologic inhibition of preterm labour. Clin Obstet Gynecol 45: 99-113
Eine orale Tokolyse mit $\beta$-Mimetika oder Magnesium erscheint als nicht sinnvoll

Zusätzlich zur Tokolyse sollte ein einzelner Zyklus Betamethason zur Induktion der fetalen Lungenreife verabreicht werden

Bei stationärer Behandlung ist auch eine engmaschige fetale Überwachung zu empfehlen
11. Kenyon SL, Taylor DJ, Tarnow-Mordi W (2001) Broad spectrum antibiotics for preterm, prelabour rupture of fetal membranes: the ORACLE I randomised trial. ORACLE collaborative group. Lancet 357: 979-988

12. Kenyon SL, Taylor DJ, Tarnow-Mordi W (2001) Broad spectrum antibiotics for spontaneous preterm labour: The ORACLE II randomised trial. Lancet 357: 989-994

13. King J, Flenady V (2000) Antibiotics for preterm labour with intact membranes. Cochrane Database Syst Rev 2: CD 000246

14. Meis PJ, Aleman A (2004) Progesterone treatment to prevent preterm birth. Drugs 64: 2463-2474

15. Moise KJ (1993) Effect of advancing gestational age on the frequency of fetel ductal constriction in association with maternal indomethacin use. Am J Obstet Gynecol 168: 1350 1353

16. Norton ME, Merill J, Cooper BA et al. (1993) Neonatal complications after the administration of indomethacin for preterm labor. N Engl J Med 329: 1602-1607 
17. Romero R, Sibai BM, Sanchez-Ramos L et al. (2000) An oxytocin reseptor antagonist (atosiban) in the treatment of preterm labor: a rando mized, double-blind, placebo-controlled trial with tocolytic rescue. Am J Obstet Gynecol 182: 1173

18. Sanchez-Ramos L, Kaunitz Am, Delke I (2005) Progestational agents to prevent preterm birth: a meta-analysis of randomized controlled trials. Obstet Gynecol 105: 273-279
19. Valenzuela GJ, Sanchez-Ramos L, Romero R et al. (2000) Maintenance treatment with the oxytocin antagonist atosiban. The atosiban PTL-098 study group. Am J Obstet Gynecol 182: 1184-1190

20. Van Veen AJ, Pelinck MJ, Pampus MG van, Erwich JJHM (2004) Severe hypotension and fetal death due to tocolysis with nifedipine. Br J Obstet Gynaecol 111: 1-2
21. The Worldwide Atosiban Versus Beta-agonists Study group (2001) Effectiveness and safety of the oxytocin antagonist atosiban versus betaadrenergic agonists in the treatment of preterm labour. Br J Obstet Gynaecol 108: 133-142

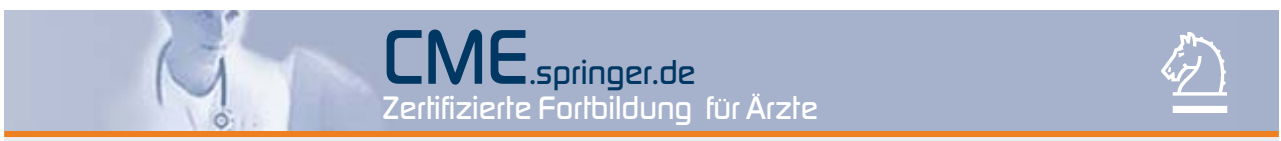

\section{Zertifizierte Fortbildung für alle mit den CME.Tickets!}

Im CME.Center auf CME.springer.de stehen Ihnen online über 250 aktuelle und zertifizierte Fortbildungseinheiten aus über 28 Bereichen der Medizin zur Verfügung. Die CME.Tickets erlauben Ihnen, das komplette CME-Angebot zu nutzen unabhängig von einem Zeitschriftenabonnement oder auch zusätzlich zum Abonnement.

Wer profitiert von diesem Angebot? Jeder Arzt - ob niedergelassen oder in der Klinik tätig - kann auf CME.springer.de einfach und qualitätsgesichert CME-Punkte sammeln. Mit den CME.Tickets können beispielsweise auch Ehepartner oder die Mitglieder einer Gemeinschaftspraxis an den Fortbildungseinheiten auf CME.springer.de teilnehmen.
Haben Sie bereits eine Springer Fachzeitschrift mit Zertifizierter Fortbildung abonniert? In diesem Fall ist die Teilnahme an den Fortbildungseinheiten Ihrer Zeitschrift für Sie bereits im Abonnementpreis enthalten.

\author{
Punkten Sie online! \\ Weitere Informationen unter \\ CME.springer.de oder bei unserem Helpdesk \\ unter CME@springer.com
}

CME.springer.de 


\section{CME}

Bitte beachten Sie:

Antwortmöglichkeit nur online unter: CME.springer.de

Die Frage-Antwort-Kombinationen werden online

CME-Fragebogen

individuell zusammengestellt.

Es ist immer nur eine Antwort möglich.

\section{Welche Untersuchung vor Durchführung einer medikamentösen Tokolyse braucht nicht routinemäßig durchgeführt zu werden? \\ $\square$ Kardiotokographie zur Be- urteilung der Häufigkeit von Kontraktionen und des fetalen Zustandes. \\ $\square$ Spekulumuntersuchung mit Bestimmung des vaginalen pH-Wertes, bakteriologischen Abstrichen einschließlich Chla- mydienabstrich bzw. PCR aus Urin und Beurteilung der Zer- vixlänge. \\ $\square$ Vaginalsonographische Mes- sung der Zervixlänge. \\ $\square$ Röntgenuntersuchung des Thorax zum Ausschluss einer Herz-Lungen-Erkrankung. \\ $\square$ Ausschluss einer systemischen Infektion durch klinische Un- tersuchung, Temperaturmes- sung, Urinstix und Laborunter- suchungen (Leukozytenzahl, CRP-Konzentration).}

\section{Welches ist kein Kriterium relevanter vorzeitiger Wehen? \\ $\square$ Zervixverkürzung \\ $\square$ Uteruskontraktionen mit einer Frequenz von $1 /$ Stunde \\ $\square$ Positiver Fibronektintest \\ $\square$ Muttermunderöffnung \\ $\square$ Schmerzhaftigkeit der Kon- traktionen}

\author{
Wann ist eine kurzfristige \\ Tokolyse angezeigt? \\ $\square$ Bei fetaler Wachstumsretardie- \\ rung \\ $\square$ Bei vorzeitigen Wehen in Ver- \\ bindung mit einer Zervixver- \\ kürzung \\ $\square$ Bei Muttermunderöffnung \\ und Zeichen der Amnioninfek- \\ tion \\ $\square$ Bei Wehen bei 36 SSW \\ $\square$ Bei vorzeitigen Wehen und \\ schwerer Präeklampsie
}

Welches Tokolytikum hat die geringsten Nebenwirkungen für Mutter und Kind?

$\square$ Atosiban

$\square$ Fenoterol

$\square$ Magnesium

$\square$ Nifedipin

Indometacin

Welches Tokolytikum steht auf der Liste der „essential medications" der WHO aus dem Jahre 2005?
$\square$ Atosiban
$\square$ Fenoterol
$\square$ Magnesium
$\square$ Nifedipin
$\square$ Indometacin

Welche Aussage trifft zu?

$\square$ Falls ein Tokolytikum allein nicht ausreicht, muss eine Kombination von Tokolytika eingesetzt werden.

$\square$ Bei Gestationsdiabetes sollte zur Tokolyse eher Atosiban als Fenoterol verwendet werden.

$\square$ Falls eine i.v.-Tokolyse nach zwei Tagen nicht zum Sistieren der Wehen führt, sollte sie oral fortgeführt werden.

$\square$ Die orale Magnesiumgabe verbessert nachgewiesenermaBen die Tokolyse mit Atosiban.

$\square$ Falls ein Tokolytikum nicht wirksam ist, sollte ein anderes Tokolytikum eingesetzt werden.

\section{Fenoterol kann in folgender Situation zur Tokolyse einge- setzt werden \\ $\square$ Bei maternalem Herzfehler \\ $\square$ Bei maternaler Hypertonie \\ $\square$ Bei maternaler Hypotonie \\ $\square$ Bei schlecht eingestelltem Dia- betes mellitus der Mutter \\ $\square$ Bei maternaler Hyperthyreose}

\section{Wann ist eine Tokolyse in der} Regel indiziert?

$\square$ Bei Amnioninfektionssyndrom

$\square$ Routinemäßig bei Drillingen ab einem Schwangerschaftsalter von 28 SSW

$\square$ Bei Zervixverkürzung ohne Wehen

$\square$ Bei vorzeitiger Lösung der Plazenta

$\square$ Bei Kontraktionen und Blutung bei Placenta praevia bei einem Schwangerschaftsalter $<28$ SSW

\section{Welche Substanz(-gruppe) besitzt keine tokolytische Wirksamkeit? \\ $\square$ Oxytocinrezeptor-Antagonis- ten \\ Alkohol \\ $\square$ Kalziumantagonisten \\ $\square$ B-Blocker \\ $\square$ Zyklooxygenaseinhibitoren}

\section{Welches ist eine sinnvolle} routinemäßige additive Maßnahme zur Tokolyse?

$\square$ Bettruhe

$\square$ Blutvolumenexpansion

$\square$ Durchführung der Lungenreifeinduktion mit Kortikosteroiden

$\square$ Antibiotikatherapie

$\square$ Gestagenverabreichung
Diese Fortbildungseinheit ist 12 Monate auf CME.springer. de verfügbar.

Den genauen Einsendeschluss erfahren Sie unter CME.springer.de 